\title{
Analysis of a Vaccination Model For Carrier Dependent Population With A Saturated Incidence Rate
}

\author{
${ }^{1}$ S. K. Tiwari, ${ }^{2}$ V. K. Gupta, ${ }^{1 *}$ Lakhan Nagar, ${ }^{3}$ Shivram Sharma \\ ${ }^{1}$ School of Studies in Mathematics, Vikram University, Ujjain (M.P.), India \\ ${ }^{2}$ Department of Mathematics, Govt. Madhav Science P.G. College, Ujjain (M.P.), India \\ ${ }^{3}$ Department of Mathematics, Medi-Caps University, Indore (M.P.), India \\ *Corresponding author: lakhannagar988@gmail.com, mob.9584314649
}

Available online at: www.isroset.org

Accepted: 10/Aug/2018, Online: 30/Aug/2018

\begin{abstract}
This study to proposed and analyzed a vaccination model for carrier dependent population with a saturated incidence rate. The equilibrium's for the model are found and their stability investigated. Gives the numerical examples in support of results
\end{abstract}

Keywords: carrier dependent, vaccination, environmental discharge, infectious diseases, carrying capacity.

\section{INTRODUCTION}

Infectious diseases are spread by carriers which are present in the environment [3]. Carriers are individuals who are able to transmit the disease but do not show any symptoms. Infectious diseases are also known as transmissible disease or communicable disease. Infectious disease can be caused by bacteria, viruses, fungi or parasites. Some infectious diseases can be passed from person to person and some are transmitted by bites from insects or animals. Infectious diseases are also transmitted by contaminated food or water. The spread of such diseases is very much dependent on the carrier population, the density of which increases due to environmental factors such as temperature, humidity, rain, vegetation, etc [13, 15]. The per capita growth rate and the modified carrying capacity of carrier population are taken to be functions of human population density and assumed to increase as the human population density increases [10,11]. Many infectious diseases are prevented by vaccination. The modeling and analysis of infectious disease have been done by many researchers [2, 4, 6, 8, 12, 14]. Infectious diseases model with population dependent death rate and logistic population growth studied by Greenhalth [1] and Gao et al. [7] . Zhou and Hethcote [5] introduced the various kinds of demographics for infectious diseases. Ghosh et al. [9] studied the spread of carrier dependent infectious diseases with environmental effects using variable carrier population.

Naresh et al. [16] considered a following vaccination model for carrier dependent infectious diseases with environmental effects:

$$
\left.\begin{array}{l}
\frac{d X}{d t}=A-\beta X Y-\lambda X C+v Y-\varphi X-d X \\
\frac{d Y}{d t}=\beta X Y+\lambda X C-(v+\alpha+d) Y+\gamma V Y+v_{1} V C \\
\frac{d V}{d t}=\varphi X-\gamma V Y-v_{1} V C-d V \\
\frac{d N}{d t}=A-d N-\alpha Y \\
\frac{d C}{d t}=s(N) C-\frac{s_{0} C^{2}}{L(N)}-s_{1} C
\end{array}\right\}
$$


with

$$
\begin{aligned}
& X+Y+V=N \\
& X(0)=X_{0}>0, Y(0)=Y_{0} \geq 0, V(0)=V_{0} \geq 0 \\
& N(0)=N_{0}>0, C(0)=C_{0} \geq 0
\end{aligned}
$$

where $N(t)$ be the total human population at any time t, which is divided into three subclasses: the susceptible $X(t)$, the infectives $Y(t)$, and the vaccinated individuals $V(t) . C(t)$ represent the density of carrier population, which is governed by a generalized logistic model. It is further assumed that the susceptible are vaccinated at a constant rate and some of them may again become infected while coming in contact with infectives or with carriers due to inefficacy of vaccines. $\beta$ and $\lambda$ are transmission coefficients due to infectives and carrier population respectively. The parameters $\phi, v$ and $\mathrm{d}$ represent vaccination coverage, therapeutic treatment coverage and natural deaths respectively, $\alpha$ is the disease related death constant, $\gamma$ and $v_{1}$ denote the transmission coefficient of vaccinated individuals due to interaction with infectives and carrier population respectively. However, the rate with which vaccinated persons become infected is very small as compared to the rate with which susceptible get infected i.e. $\gamma \leq \beta$ and $v_{1} \leq \lambda$. The constant $s_{1}$ is the death rate coefficient of carriers due to natural factors as well as by control measures. Here, $s(N)$ denotes growth rate per capita of the carrier population density. It may be noted that if the growth rate and death rate due to natural as well as control measure of carrier population are balanced, then it may tend to zero. Similarly, $L(N)$ is the modified carrying capacity of the carrier population. It has been pointed out in the introduction, that as the human population increases, the effects of human population related factors enhance the changes of growth of carrier population.

\subsection{The model}

In this study, consider a saturated incidence rate $\frac{\beta X Y}{1+a Y}$, where $a$ is the psychological effect rate. The model dynamics is governed by following system of nonlinear ordinary differential equations:

$$
\begin{aligned}
& \frac{d X}{d t}=A-\frac{\beta X Y}{1+a Y}-\lambda X C+v Y-\varphi X-d X \\
& \frac{d Y}{d t}=\frac{\beta X Y}{1+a y}+\lambda X C-(v+\alpha+d) Y+\frac{\gamma V Y}{1+a Y}+v_{1} V C \\
& \frac{d V}{d t}=\varphi X-\frac{\gamma V Y}{1+a Y}-v_{1} V C-d V \\
& \frac{d N}{d t}=A-d N-\alpha Y \\
& \frac{d C}{d t}=s(N) C-\frac{s_{0} C^{2}}{L(N)}-s_{1} C \\
& X+Y+V=N \\
& X(0)=X_{0}>0, Y(0)=Y_{0} \geq 0, V(0)=V_{0} \geq 0 \\
& N(0)=N_{0}>0, C(0)=C_{0} \geq 0
\end{aligned}
$$

where $a$ is the psychological effect rate and rest of the parameters are describes in above model (*). Thus, in the model (1), $s(N)$ and $L(N)$ are taken to be functions of total human population instead of infective population. Assume that the growth rate per capita increase as the human population density increase, we have 
$s(0)=s_{0}$ and $s^{\prime}(N) \geq 0$,

where $s_{0}$ is the value of $s(N)$ at $N=0$ and ()$^{\prime}$ denotes the derivatives of the function with respect to its argument. We also assume that the modified carrying capacity increase with human population density, so that

$L(0)=L_{0}>0$ and $L^{\prime}(N) \geq 0$,

where $L_{0}$ is the value of $L(N)$ when $N=0$ From equation $(1),(2)$ and(3), we see that even if human population related factors are absent, carrier population density increase in its natural environment and it tends to $L_{0}\left(1-\frac{s_{1}}{s_{0}}\right)$ which may become zero if $s_{1} \rightarrow s_{0}$. In the model, all the dependent variables and parameters are assumed to be non negative.

\section{EQUILIBRIUM ANALYSIS}

$$
\left.\begin{array}{rl}
\frac{d Y}{d t} & =\frac{\beta Y(N-Y-V)}{1+a Y}+\lambda C(N-Y-V)-(v+\alpha+d) Y+\frac{\gamma V Y}{1+a Y}+v_{1} V C, \\
\frac{d V}{d t} & =\varphi(N-Y-V)-\frac{\gamma V Y}{1+a Y}-v_{1} V C-d V, \\
\frac{d N}{d t} & =A-d N-\alpha Y, \\
\frac{d C}{d t} & =s(N) C-\frac{s_{0} C^{2}}{L(N)}-s_{1} C
\end{array}\right\}
$$

There exist following three non negative equilibria of the system (4)

1. Disease free equilibrium $E_{0}=\left(0, \frac{\varphi A}{d(d+\varphi)}, \frac{A}{d}, 0\right)$ exists, without any condition. The $\quad$ existence of $E_{0}$ is obvious.

2. Carrier free equilibrium $E_{1}=(\bar{Y}, \bar{V}, \bar{N}, 0)$.

This equilibrium may be obtain by solving the following algebraic equations,

$$
\begin{aligned}
& \beta(N-Y)-(v+\alpha+d)(1+a Y)-(\beta-\gamma) V=0 \\
& Y=\frac{A-d N}{\alpha} \\
& V=\frac{\varphi\left[1+a\left(\frac{A-d N}{\alpha}\right)\right][(\alpha+d) N-A]}{\alpha(\varphi+d)\left[1+a\left(\frac{A-d N}{\alpha}\right)\right]+\gamma(A-d N)}
\end{aligned}
$$

Using equation (6) and (7) in equation (5), we get an algebraic equation in single variable $N$. i.e. $F(N)=0$ where $F(N)=0$ is given by the following equation.

$$
F(N)=[\beta N-(v+\alpha+d)(1+a Y)]-(\beta-\gamma) V-\beta Y=0
$$


From equation (6) and (7), we note that $\bar{Y}$ and $\bar{V}$ will be positive only when $F(N)=0$ has a root in the interval $\left(\frac{A}{\alpha+d}, \frac{A}{d}\right)$. From equation (8) it is easy to observe that, $F\left(\frac{A}{\alpha+d}\right)<0$ and $F\left(\frac{A}{d}\right)>0$, if $\frac{A(\beta d+\gamma \varphi)}{d(\phi+d)(v+\alpha+d)}>1$ , thus there exists a root $\bar{N}$ of $F(N)=0$ in $\frac{A}{\alpha+d}<N<\frac{A}{d}$. Also $F^{\prime}(N)>0$ in $\frac{A}{\alpha+d}<N<\frac{A}{d}$.hence there exists a unique positive root $\bar{N}$ given by $F(N)=0$. Knowing the value of $\bar{N}$, we can compute the values of $\bar{Y}$ and $\bar{V}$ from equations (6) and (7), respectively.

Thus there exists a unique carrier free equilibrium $E_{1}(\bar{Y}, \bar{V}, \bar{N}, 0), \quad$ provided the condition $R(\varphi)=\frac{A(\beta d+\gamma \varphi)}{d(\phi+d)(v+\alpha+d)}>1$ is satisfied.

\subsection{Endemic equilibrium}

The endemic equilibrium, $E_{2}=\left(Y^{*}, V^{*}, N^{*}, C^{*}\right)$

The endemic equilibrium is given by the solution of the following set of algebraic equations,

$$
\begin{aligned}
& \left(\beta Y+\lambda c(1+a Y)(N-Y-V)+\left(\gamma Y+v_{1} C(1+a Y) V\right)-(v+\alpha+d) Y(1+a Y)=0\right. \\
& Y=\frac{A-d N}{\alpha} \\
& V=\frac{\varphi((\alpha+d) N-A)(1+a Y)}{\alpha\left[\left(\varphi+d+v_{1} C\right)(1+a Y)\right]+\gamma(A-d N)} \\
& C=\frac{L(N)\left[s(N)-s_{1}\right]}{s_{0}}
\end{aligned}
$$

We may reduce equation (9) in a single variable $N$ i.e. $F(N)$ by using equations (10),(11) and (12), where

$$
F(N)=\left(\beta Y+\lambda c(1+a Y)(N-Y-V)+\left(\gamma Y+v_{1} C(1+a Y) V\right)-(v+\alpha+d) Y(1+a Y)\right.
$$

It is clear from (13) that $F\left(\frac{A}{\alpha+d}\right)<0$ and $F\left(\frac{A}{d}\right)>0$.this implies that there exists a root $\mathrm{N}$ of $\mathrm{F}(\mathrm{N})=0$ in $\frac{A}{\alpha+d}<N<\frac{A}{d}$. Also $F^{\prime}(N)>0$, provided $\left[\alpha v_{1} N C^{\prime}-\gamma A\right]>0$. Hence there exist a unique positive root $N^{*}$ given by $\mathrm{F}(\mathrm{N})=0$ in $\frac{A}{\alpha+d}<N<\frac{A}{d}$. Knowing the value of $N^{*}$, the values of $Y^{*}, V^{*}$ and $C^{*}$ can be computed from equations (10),(11)and (12), respectively. Thus, the equilibrium $E_{2}$ exists provided $s\left(N^{*}\right)-s_{1}>0$ and $F^{\prime}(N)>0$. From equation (13) it is easy to note that $F(\bar{N})>0$ keeping in mind the above analysis of equation (13), we have $\bar{N}>N^{*}$. From equation (12), it may be noted that $d C^{*} / d N^{*}>0$ in view of equation (2) and (3). Thus, the equilibrium value of carrier population density increases with increase in the equilibrium value of human population. 


\section{STABILITY ANALYSIS}

Now we analyze the stability of equilibria $E_{o}, E_{1}$ and $E_{2}$. The local stability results of these equilibria are stated in the following theorem:

Theorem 3.1. The equilibrium $E_{0}$ is unstable whenever $E_{1}$ or $E_{2}$ exists, $E_{1}$ is unstable whenever $E_{2}$ exists and the equilibrium $E_{0}$ and $E_{0}$ are locally asymptotically stable provided the following conditions are satisfied.

$$
\begin{aligned}
\alpha\left[\frac{(\beta-\gamma) Y^{*}}{\left(1+a Y^{*}\right)}+\left(\lambda-v_{1}\right) C^{*}\right]^{2}<\frac{1}{4} b_{1} b_{2}{ }^{2} b_{3} \min \left\{\frac{b_{1}}{4 b_{3}\left(\varphi+\frac{\gamma V^{*}}{\left(1+Y^{*}\right)}\right)^{2}}, \frac{d}{3(\varphi)}, \frac{k_{3} s_{0} C^{*}}{3 v_{1}^{2} V^{* 2} L\left(N^{*}\right)}\right\} \\
3 \alpha L^{2}\left(N^{*}\right)\left[\lambda\left(N^{*}-Y^{*}-V^{*}\right)+v_{1} V^{*}\right]^{2}\left[s^{\prime}\left(N^{*}\right)+\frac{s_{0} C^{*} L^{\prime}\left(N^{*}\right)}{L^{2}\left(N^{*}\right)}\right]^{2}<\frac{8}{27} b_{1} b_{3} d s_{0}{ }^{2} \\
b_{1}=\frac{\lambda N^{*} C^{*}}{Y^{*}}-\frac{\left(\lambda-v_{1}\right) V^{*} C^{*}}{Y^{*}}+\frac{\beta}{\left(1+a Y^{*}\right)^{2}} \\
b_{2}=\varphi+d+\frac{\gamma Y^{*}}{\left(1+a Y^{*}\right)}+v_{1} C^{*} \\
b_{3}=\frac{\beta Y^{*}}{\left(1+a Y^{*}\right)}+\lambda C^{*}
\end{aligned}
$$

Proof: the general variational matrix $\mathrm{M}$ for the system is given as follows:

$$
M=\left(\begin{array}{cccc}
m_{11} & m_{12} & m_{13} & m_{14} \\
-\phi+\frac{(1+a Y) \gamma V+\gamma V Y a}{(1+a Y)^{2}} & -\phi-\frac{\gamma Y}{(1+a Y)}-v_{1} C-d & \phi & -v_{1} V \\
-\alpha & 0 & -d & 0 \\
0 & 0 & s^{\prime}(N) C+\frac{s_{0} C^{2}}{L^{2}(N)} L^{\prime}(N) & s(N)-s_{1}-\frac{2 s_{0} C}{L(N)}
\end{array}\right)
$$

where

$$
\begin{aligned}
& m_{11}=(1+a Y)(\beta N-2 \beta Y-\beta V+\gamma V)-\left(\beta Y N-\beta Y^{2}-\beta V Y+\gamma V Y\right) a-\lambda C-(v+\alpha+d) \\
& m_{12}=-\frac{\beta Y}{1+a Y}-\lambda C+\frac{\gamma Y}{1+a Y}+v_{1} C \\
& m_{13}=\frac{\beta Y}{1+a Y}+\lambda C \\
& m_{14}=\lambda(N-Y-V)+v_{1} V
\end{aligned}
$$

At $E_{0}$ 


$$
\begin{gathered}
M_{0}=\left(\begin{array}{cccc}
\frac{A}{d(\phi+d)}(\beta d+\gamma \phi)-(v+\alpha+d) & 0 & 0 & \frac{\lambda A d+v_{1} \phi A}{d(\phi+d)} \\
-\phi+\frac{\gamma \phi A}{d(\phi+d)} & -(\phi+d) & \phi & \frac{-v_{1} \phi A}{d(\phi+d)} \\
-\alpha & 0 & -d & 0 \\
0 & 0 & 0 & s\left(\frac{A}{d}\right)-s_{1}
\end{array}\right) \\
\psi_{1}=s\left(\frac{A}{d}\right)-s_{1} \\
\psi_{2}=\frac{A}{d(\varphi+d)}(\beta d+\gamma \varphi)-(v+\alpha+d) \\
\psi_{3}=-d \\
\psi_{4}=-(\varphi+d)
\end{gathered}
$$

Since all the model parameters are assumed to be non negative, it follows that $\psi_{3} \cdot \psi_{4}<0$. Thus, the stability of $E_{0}$ will depend on the sign of $\psi_{1}$ and $\psi_{2}$.

We define a threshold parameter $R(\varphi)=\frac{A(\beta d+\gamma \varphi)}{d(\varphi+d)(v+\alpha+d)}$

The disease free equilibrium is locally asymptotically stable if $R(\phi)<1$ and $s\left(\frac{A}{d}\right)-s_{1}<0$. Since $s(N)$ is increasing function of $N$, so $\psi_{1}<0$, this always implies that $s(N)-s_{1}<0$. biologically $s\left(\frac{A}{d}\right)-s_{1}<0$ implies the absence of carrier population. Thus DFE may be stable only in the absence of carrier population because otherwise disease still persists even if there is no direct interaction of susceptible with infectives. Thus, $E_{0}$ is unstable if either $R(\phi)>1$ or $s\left(\frac{A}{d}\right)-s_{1}>0$, keeping in mind that the necessary condition for the existence of $E_{1}$ is $R(\phi)>1$ and for the existence of $E_{2}$ is $s\left(N^{*}\right)-s_{1}>0$. Thus, $E_{0}$ is unstable whenever $E_{1}$ or $E_{2}$ exists, similarly one of the eigen values of the variational matrix is $s(\bar{N})-s_{1}$. Now keeping in mind that $\bar{N}>N^{*}$, we have that $E_{1}$ is unstable whenever $E_{2}$ is exists.

From equation (5), (6) and (7), we get $\phi$ as a function of Y alone

$$
\phi(Y)=\frac{\left[\beta Y-\frac{\beta(A-\alpha Y)}{d}+(v+\alpha+d)(1+a Y)\right][d(1+a Y)+\gamma Y]}{(1+a Y)\left[-(v+\alpha+d)(1+a Y)+\frac{\gamma(A-\alpha Y)}{d}-\gamma Y\right]}
$$

We note that, 


$$
\begin{aligned}
\phi(0) & =d\left[\frac{\frac{\beta A}{d(v+\alpha+d)}-1}{1-\frac{\gamma A}{d}(v+\alpha+d)}\right] \\
& =d\left[\frac{R_{0}-1}{1-R_{1}}\right]
\end{aligned}
$$

The expression for $\phi(0)$ is the threshold vaccination rate given by the term $\phi c_{1}$. We have already shown the uniqueness of $\bar{Y}$ for $R(\phi)>1$ in section 3, Now here we show the bifurcation analysis for the disease free equilibrium $E_{0}$ when $R(\phi)=1$. We note that for $R(\phi)=1$, one eigenvalue of $M_{0}$ is 0 , whereas other eigen values are negative provided $s(A / d)-s_{1}<0$.

\section{Local Stability of endemic equilibrium}

$$
\begin{gathered}
\begin{array}{l}
U_{1}=\frac{1}{2}\left(k_{0} y^{2}+k_{1} v^{2}+k_{2} n^{2}+k_{3} c^{2}\right) \\
Y=Y^{*}+y \\
V=V^{*}+v \\
N=N^{*}+n \\
C=C^{*}+c
\end{array} \\
\begin{array}{l}
\frac{d U_{1}}{d t}=-k_{0}\left[\frac{\beta}{\left(1+a Y^{*}\right)^{2}}+\frac{\lambda N^{*} C^{*}}{Y^{*}}+\frac{\left(\lambda-v_{1}\right) V^{*} C^{*}}{Y^{*}}\right] y^{2}-k_{1}\left(\varphi+d+\frac{\gamma Y^{*}}{1+a Y^{*}}+v_{1} C^{*}\right) v^{2} \\
-k_{2} d n^{2}-\frac{k_{3} s_{0} C^{*}}{L\left(N^{*}\right)} c^{2}-\left[k_{0}\left\{\frac{(\beta-\gamma) Y^{*}}{1+a Y^{*}}+\left(\lambda-v_{1}\right) C^{*}\right\}+k_{1}\left(\varphi+\frac{\gamma V^{*}}{\left(1+a Y^{*}\right)^{2}}\right)\right] v y \\
+k_{1} \varphi v n-k_{1} v_{1} V^{*} v c+k_{0}\left[\left(\frac{\beta Y^{*}}{1+a Y^{*}}+\lambda C^{*}\right)-\alpha k_{2}\right] n y+k_{0}\left[\lambda\left(N^{*}-Y^{*}-V^{*}\right)+v_{1} V^{*}\right] y c \\
+k_{3}\left[s^{\prime}\left(N^{*}\right) C^{*}+\frac{s_{0} C^{* 2}}{L^{2}\left(N^{*}\right)} L^{\prime}\left(N^{*}\right)\right] n c
\end{array}
\end{gathered}
$$

$\frac{d U_{1}}{d t}$ will be negative under the following conditions

$d t$

$$
\begin{aligned}
k_{0}\left[(\beta-\gamma) \frac{Y^{*}}{1+a Y^{*}}+\left(\lambda-v_{1}\right) C^{*}\right]^{2}<k_{1}\left(\varphi+d+\frac{\gamma Y^{*}}{1+a Y^{*}}+v_{1} C^{*}\right)\left[\frac{\lambda N^{*} C^{*}}{Y^{*}}-\frac{\left(\lambda-v_{1}\right) V^{*} C^{*}}{Y^{*}}+\frac{\beta}{\left(1+a Y^{*}\right)^{2}}\right] \\
k_{1}\left(\varphi+\frac{\gamma V^{*}}{\left(1+a Y^{*}\right)^{2}}\right)^{2}<k_{0}\left(\varphi+d+\frac{\gamma Y^{*}}{1+a Y^{*}}+v_{1} C^{*}\right)\left[\frac{\beta}{\left(1+a Y^{*}\right)^{2}}+\frac{\lambda N^{*} C^{*}}{Y^{*}}-\frac{\left(\lambda-v_{1}\right) V^{*} C^{*}}{Y^{*}}\right]
\end{aligned}
$$




$$
\begin{gathered}
{\left[k_{0}\left(\frac{\beta Y^{*}}{1+a Y^{*}}+\lambda C^{*}\right)-\alpha k_{2}\right]^{2}<\frac{2 k_{0} k_{2} d}{3}\left[\frac{\lambda N^{*} C^{*}}{Y^{*}}-\frac{\left(\lambda-v_{1}\right) V^{*} C^{*}}{Y^{*}}+\frac{\beta}{\left(1+a Y^{*}\right)^{2}}\right]} \\
k_{0}\left[\lambda\left(N^{*}-Y^{*}-V^{*}\right)+v_{1} V^{*}\right]^{2}<\frac{2 k_{3}}{3} \frac{s_{o} C^{*}}{L\left(N^{*}\right)}\left[\frac{\lambda N^{*} C^{*}}{Y^{*}}-\frac{\left(\lambda-v_{1}\right) V^{*} C^{*}}{Y^{*}}+\frac{\beta}{\left(1+a Y^{*}\right)^{2}}\right] \\
k_{1} \varphi^{2}<\frac{k_{2}}{3} d\left(\varphi+d+\frac{\gamma Y^{*}}{1+a Y^{*}}+v_{1} C^{*}\right) \\
k_{1} v^{2} V^{* 2}<\frac{k_{3}}{3} \frac{s_{0} C^{*}}{L\left(N^{*}\right)}\left(\varphi+d+\frac{\gamma Y^{*}}{1+a Y^{*}}+v_{1} C^{*}\right) \\
k_{3}\left[s^{\prime}(N) C^{*}+\frac{s_{0} C^{* 2}}{L^{2}\left(N^{*}\right)} L^{\prime}\left(N^{*}\right)\right]^{2}<\frac{4 k_{2} d s_{0} C^{*}}{9 L\left(N^{*}\right)}
\end{gathered}
$$

We choose $k_{1}$ and $k_{3}$ such that

$$
\begin{gathered}
\frac{4 \alpha}{b_{1} b_{2} b_{3}}\left[\frac{(\beta-\gamma) Y^{*}}{1+a Y^{*}}+\left(\lambda-v_{1}\right) C^{*}\right]^{2}<k_{1}<b_{2} \min \left\{\frac{b_{1}}{4 b_{3}\left(\varphi+\frac{\gamma V^{*}}{\left(1+a Y^{*}\right)^{2}}\right)}, \frac{d}{3 \varphi}, \frac{k_{3} s_{0} C^{*}}{3 v_{1}^{2} V^{* 2} L\left(N^{*}\right)}\right\} \\
\frac{3 \alpha L\left(N^{*}\right)}{2 s_{0} C^{*} b_{1} b_{3}}\left[\lambda\left(N^{*}-Y^{*}-V^{*}\right)+v_{1} V^{*}\right]^{2}<k_{3}<\frac{4 d s_{0} C^{*}}{9 L\left(N^{*}\right)\left[s^{\prime}\left(N^{*}\right) C^{*}+\frac{s_{0} C^{* 2}}{L^{2}\left(N^{*}\right)} L^{\prime}\left(N^{*}\right)\right]} \\
b_{1}=\frac{\lambda N^{*} C^{*}}{Y^{*}}-\frac{\left(\left(\lambda-v_{1}\right) V^{*} C^{*}\right.}{Y^{*}}+\frac{\beta}{\left(1+a Y^{*}\right)^{2}} \\
b_{2}=\varphi+d+\frac{\gamma Y^{*}}{1+a Y^{*}}+v_{1} C^{*} \\
b_{3}=\frac{\beta Y^{*}}{1+a Y^{*}}+\lambda C^{*}
\end{gathered}
$$

Hence $\frac{d U_{1}}{d t}$ is a negative definite under the conditions as stated in the statement of the theorem, showing that $E_{2}$ is locally asymptotically stable.

To study the non linear asymptotic stability of endemic equilibrium $E_{2}$, we require the bounds of dependent variables. For this, we state the following lemma giving the region of attraction, with out proof.

Lemma 3.2. the region of attraction for the system is given by,

$$
\Omega=\left\{(Y, N, V, C): 0 \leq Y \leq N \leq \frac{A}{d}, 0 \leq V \leq \frac{\varphi A}{d(\varphi+d)}, 0 \leq C \leq C_{m}\right\}
$$


Which attracts all the solutions initiating in the positive orthant, where $C_{m}=\frac{L(A / d)}{s_{0}}\left[s(A / d)-s_{1}\right]$

Theorem 3.3. In addition to assumptions (2) and (3), let $s(N)$ and $L(N)$ satisfy $0 \leq s^{\prime}(N) \leq p$ and $0 \leq L^{\prime}(N) \leq q$ for some positive constant $p$ and $q$ in $\Omega$, then $E_{2}$ is nonlinearly asymptotically stable in $\Omega$ provided the following inequalities are satisfied:

$$
\alpha N^{*}\left[\frac{\beta-\gamma}{N^{*}}+\frac{\lambda C_{m}}{Y^{*}}\right]<\frac{1}{5} d \beta,
$$

$\alpha N^{*} L^{2}\left(N^{*}\right)\left[\lambda\left(N^{*}-Y^{*}-V^{*}\right)+v_{1} V^{*}\right]^{2}\left[p+\frac{s_{0} q C_{m}}{L_{0}^{2}}\right]^{2}<\frac{4}{45} \beta d s_{0}{ }^{2} Y^{* 2}$,

$$
N^{*}\left[\frac{\beta-\gamma}{N^{*}}+\frac{\left(\lambda-v_{1}\right) C_{m}}{Y^{*}}\right]^{2}<\frac{1}{5} \beta\left(\varphi+d+\frac{\gamma Y^{*}}{N^{*}}\right)^{2} \times
$$

$$
\min \left\{\frac{\beta}{5\left(\varphi+\frac{\gamma \varphi A}{d(\varphi+d) N^{*}}\right)^{2}}, \frac{d}{4\left(\varphi+\frac{\gamma A}{d N^{*}}\right)^{2}}, \frac{m_{3} s_{0}}{3 v_{1}^{2} V^{* 2} L\left(N^{*}\right)}\right\}
$$

it is clear from (19) that in the absence of human related factors, i.e. $p=q=0$, the inequality is automatically satisfied. This implies that human population related factors, conductive to the growth of carrier population, have a destabilizing effect on the system. Here we also note that due to presence of a vaccinated class, a condition (20) is required for the nonlinear stability which further destabilizes the system.

\section{Proof.}

$$
\begin{aligned}
& U_{2}=m_{0}\left(Y-Y^{*}-Y^{*} \ln \frac{Y}{Y^{*}}\right)+\frac{m_{1}}{2}\left(V-V^{*}\right)^{2}+\frac{m_{2}}{2}\left(N-N^{*}\right)^{2}+m_{3}\left(C-C^{*}-C^{*} \ln \frac{C}{C^{*}}\right) \\
& \frac{d U_{2}}{d t}=-\left[\frac{m_{0} \lambda N C}{Y Y^{*}}+m_{0} \frac{\left(\lambda-v_{1}\right) V C}{Y Y^{*}}\right]\left(Y-Y^{*}\right)^{2}-m_{1} v_{1} C\left(V-V^{*}\right)^{2} \\
& -\left\{\frac{m_{0} \beta}{5(1+a Y) N^{*}}\left(Y-Y^{*}\right)^{2}+\frac{m_{2} d}{4}\left(N-N^{*}\right)^{2}\right\}-m_{0}\left[\frac{\lambda C}{Y^{*}}+\frac{\beta(Y+V)}{(1+a Y) N^{*}}-\frac{\gamma V}{(1+a Y) N^{*}}\right]\left(Y-Y^{*}\right)\left(N-N^{*}\right) \\
& -\left\{\frac{m_{0} \beta}{5(1+a Y) N^{*}}\left(Y-Y^{*}\right)^{2}+\frac{m_{2} d}{4}\left(N-N^{*}\right)^{2}+m_{2} \alpha\left(Y-Y^{*}\right)\left(N-N^{*}\right)\right\} \\
& -\left\{\frac{m_{0} \beta}{5(1+a Y) N^{*}}\left(Y-Y^{*}\right)^{2}+\frac{m_{1}}{4}\left(\varphi+d+\frac{\gamma Y^{*}}{\left(1+a Y^{*}\right)}\right)\left(V-V^{*}\right)^{2}\right\}-m_{0}\left[\frac{\left(\lambda-v_{1}\right) C}{Y^{*}}+\frac{(\beta-\gamma)}{\left(1+a Y^{*}\right)}\right]\left(Y-Y^{*}\right)\left(V-V^{*}\right) \\
& -\left\{\frac{m_{o} \beta}{5(1+a Y) N^{*}}\left(Y-Y^{*}\right)^{2}+\frac{m_{1}}{4}\left(\varphi+d+\frac{\gamma Y^{*}}{\left(1+a Y^{*}\right)}\right)\left(V-V^{*}\right)^{2}+m_{1}\left(\varphi+\frac{\gamma V}{1+a Y^{*}}\right)\left(Y-Y^{*}\right)\left(V-V^{*}\right)\right\}
\end{aligned}
$$




$$
\begin{gathered}
-\left\{\frac{m_{o} \beta}{5(1+a Y) N^{*}}\left(Y-Y^{*}\right)^{2}+\frac{m_{3} s_{0}}{3 L(N)}\left(C-C^{*}\right)^{2}-m_{0} \frac{\lambda\left(N^{*}-Y^{*}-V^{*}\right)+v_{1} V^{*}}{Y^{*}}\left(Y-Y^{*}\right)\left(C-C^{*}\right)\right\} \\
-\left\{\frac{m_{1}}{4}\left(\varphi+d+\frac{\gamma Y^{*}}{1+a Y^{*}}\right)\left(V-V^{*}\right)^{2}+\frac{m_{2} d}{4}\left(N-N^{*}\right)^{2}-m_{1}\left(\varphi+\frac{\gamma V Y}{(1+a Y) N^{*}}\right)\left(V-V^{*}\right)\left(N-N^{*}\right)\right\} \\
-\left\{\frac{m_{1}}{4}\left(\varphi+d+\frac{\gamma Y^{*}}{1+a Y^{*}}\right)\left(V-V^{*}\right)^{2}+\frac{m_{3} s_{0}}{3 L(N)}\left(C-C^{*}\right)^{2}+m_{1} v_{1} V^{*}\left(V-V^{*}\right)\left(C-C^{*}\right)\right\} \\
-\left\{\frac{m_{2} d}{4}\left(N-N^{*}\right)^{2}+\frac{m_{3} s_{0}}{3 L\left(N^{*}\right)}\left(C-C^{*}\right)^{2}-m_{3}\left(f(N)+s_{0} C g(N)\right)\left(C-C^{*}\right)\left(N-N^{*}\right)\right\}
\end{gathered}
$$

where $f(N)$ and $g(N)$ are defined as follows

$$
\begin{gathered}
f(N)= \begin{cases}\frac{s(N)-s\left(N^{*}\right)}{N-N^{*}}, & N \neq N^{*} \\
\frac{d s}{d N}, & N=N^{*}\end{cases} \\
g(N)= \begin{cases}\frac{L(N)-L\left(N^{*}\right)}{\left(N-N^{*}\right) L(N) L\left(N^{*}\right)}, & N \neq N^{*} \\
\frac{1}{L^{2}\left(N^{*}\right)} \frac{d L}{d N}, & N=N^{*}\end{cases}
\end{gathered}
$$

Then by using assumptions of the theorem and the mean value theorem, we have ,

$|f(N)| \leq p$ and $|g(N)| \leq \frac{q}{L_{0}^{2}}$

After choosing $m_{0}=1, m_{2}=\frac{1}{\alpha}$ we choose $m_{1}$ and $m_{3}$ such that:

$$
\frac{N^{*} L\left(N^{*}\right)\left[\lambda(N-Y-V)+v_{1} V^{*}\right]^{2}}{s_{0} \beta Y^{* 2}}<m_{3}<\frac{4}{45} \frac{d s_{0}}{L\left(N^{*}\right)} \frac{1}{\left[p+\frac{s_{0} q C_{m}}{L_{0}^{2}}\right]^{2}}
$$

The stability conditions can then be easily obtained, as given in the statement of the theorem. Thus negative definite under the conditions (18)-(20). Hence proof.

The above theorem implies that under appropriate conditions, if the carrier population density increases, then the number of infectives in human population also increases leading to fast spread of carrier dependent infectious diseases.

\section{VACCINE INDUCED REPRODUCTION NUMBER}

We define

$$
R(\varphi)=\frac{A(\beta d+\gamma \varphi)}{d(\varphi+d)(v+\alpha+d)}=\frac{A \beta}{d(v+\alpha+d)}\left[1-\frac{(\beta-\gamma) \varphi}{\beta(\varphi+d)}\right]
$$

where 


$$
R^{\prime}(\varphi)=-\frac{A(\beta-\gamma) d}{d(\varphi+d)^{2}(v+\alpha+d)}<0 \quad(\text { since } \beta \geq \gamma)
$$

Thus $R(\varphi)$ is a decreasing function in $\varphi \geq 0$. This indicates the impact of vaccination in reducing the vaccine induced reproduction number. Moreover, in the absence of vaccination i.e.,

$$
\varphi=0, R(\varphi)=\frac{A \beta}{d(v+\alpha+d)}=R_{0}
$$

From the definition of $R(\varphi)$ and $R_{0}$, it is clear that the introduction of vaccination implies $R(\varphi) \leq R_{0}$ and consequently, if $R_{0}<1$ then $R(\varphi)<1$ when $\varphi>0$. Thus $E_{0}$ is locally asymptotically stable as long as $R(\varphi)$ is less than one.

\section{NUMERICAL SIMULATION}

Let us take the parameters $A=100, \beta=0.001, d=0.1, \varphi=0.8, v=1.8, \alpha=1.9, \lambda=2$, $\gamma=0.001, v_{1}=2$, then we obtain $R(\varphi)=0.263<1$. In this case disease dies out

Again take the parameters $A=100, \beta=0.001, d=0.0166, \varphi=0.65, v=0.9, \alpha=0.8, \lambda=2$, $\gamma=0.001, v_{1}=2$, then we obtain $R(\varphi)=3.4962$. In this case disease persist

\section{CONCLUSIONS}

In this paper, we have analyzed a vaccination model for carrier dependent population with a saturated incidence rate. We have shown that there are three non-negative equilibrium, namely disease free (DFE), carrier free (CFE) and the endemic equilibrium. The stability analysis has shown that the disease free and endemic equilibriums are locally asymptotically stable under certain condition by using Routh Hurwitz criterion.

\section{REFERENCES}

[1] D. Greenhalth, "some results for an SEIR epidemic model with density dependence in the death rate", IMA J. Math. Appl. Med Biol., 9, 67106, 1992.

[2] F. Bereszovsky, G. Karev, B. Song, C. Castillo-Chavez," A simple epidemic model with surprising dynamics", Math. Biosc. Engg., 2(1), 133$152,2005$.

[3] G.S. Harold, "Household and stored food insects of public health importance", US Department of Health, Education and Welfare, Communicable Disease Center, Atlanta, GA, 1960.

[4] H.W. Hethcote, "Qualitative analysis of communicable disease models", Math. Biosc., 28, 335-356, 1976.

[5] J. Zhou, H.W. Hethcote, "Population size dependent incidence in models for diseases without immunity", J. Math. Biol. 32, 809-834,1994.

[6] K.L. Cooke," Stability Analysis for a vector Disease model”, Rocky Mountain J. Math., 9, 31-42, 1979.

[7] L.Q. Gao, H.W. Hethcote, "Disease transmission model with density dependence demographics", J. Math. Biol. 32,717-731, 1992.

[8] L.Esteva , M. Matias, “A model for vector transmitted diseases with saturation incidence”, J. Biol. Sys., 9(4), 235-245, 2001.

[9] M. Ghosh, P. Chandra, P. Sinha, J.B. Sukla, "An Epidemiological model for carrier dependent infectious diseases with environmental effect", Int. J. Appl. Sc. Comp., 7, 188-204, 2000.

[10] M. Ghosh, P. Chandra, P. Sinha, J.B. Sukla, "Modeling the spread of carrier dependent infectious disease with environmental effects",Appl. Math. Comp., 152, 385-402, 2004.

[11] M. Ghosh, P.Chandra, P. Sinha, J.B. Sukla," Modeling the spread of bacterial disease: effect of service providers from an environmentally degraded region", Appl. Math. Comp., 160, 615-647, 2005.

[12] N.T.J. Bailey, "Spatial models in the epidemiology of infectious diseases", Lecture Notes in Biomathematics, 38,233-261,1980.

[13] P. Das, D. Mukharjee, A. K. Sarkar, "Study of carrier dependent infectious disease cholera", J. Biol. Sys., 13(3),233-244, 2005.

[14] S. Hsu, A.. Zee," Global spread of infectious diseases”, J. Bio. Sys., 12, 289-300, 2004.

[15] S. Singh, P. Chandra, J.B. Sukla, "Modelling and analysis of the spread of carrier dependent infectious diseases with environmental effects", J. Biol. Sys.,11(3), 325-335, 2003. 